\title{
Effect of lateral reinforcements on the adhesion and friction of micropillar adhesives
}

\author{
Peter van Assenbergh ${ }^{1}$ (i) $\cdot$ Kai Zhang ${ }^{1} \cdot$ Josephus G. Buijnsters ${ }^{2}$ (i) $\cdot$ Dimitra Dodou $^{1}$ (i)
}

Received: 5 May 2020 / Accepted: 27 August 2020

(c) The Author(s) 2020

\begin{abstract}
Micropillar adhesives have gained increasing attention because they generate high pull-off forces. The generation of high friction, however, has been proven difficult with such geometries, because micropillars tend to buckle under shear loading. Here, we fabricated orthogonal arrays of composite poly-dimethoxysiloxane (PDMS) micropillars with a stiff core and spincoated them with PDMS solutions to form a soft coating, as well as bridges between neighboring micropillars. We used 10 $\mathrm{wt} \%$ and $5 \mathrm{wt} \%$ PDMS solution to obtain thick or thin bridges, respectively. The micropillars had an average height of about $60 \mu \mathrm{m}$ and a diameter of $40 \mu \mathrm{m}$. Adhesion and friction measurements were performed with three types of adhesives (i.e., without bridges and with either thin or thick bridges) as well as unpatterned samples as reference, on stiff glass substrates and on deformable PDMS substrates. We found that, on PDMS substrates, bridging resulted in increased friction, compared to non-bridged micropillars. Friction increased with increasing bridge thickness, presumably due to buckling prevention. The adhesives were also subjected to 99 repeating friction cycles to test the effect of micropillar bridging on the durability of the adhesives. The results showed that adhesives with thick micropillar bridges preserved their friction performance over the cycles, whereas adhesives with no bridges or thin bridges exhibited a gradual decay of friction.
\end{abstract}

Keywords Micropillar adhesives $\cdot$ Adhesion $\cdot$ Friction $\cdot$ Reinforced adhesives

\section{Introduction}

Pressure-sensitive adhesives can be detached and reattached only a limited number of times because they tend to be gradually fouled with dust and other particles. Over the last years, gecko-inspired adhesives have been being developed as an attractive alternative of pressure-sensitive adhesives that can detach and reattach multiple times without deterioration of their adhesive performance. Opposite to pressure-sensitive adhesives that employ some type of glue, gecko adhesion is 'dry', relying on Van der Waals forces [1].

Peter van Assenbergh

s.p.vanassenbergh@tudelft.nl

$\triangle$ Kai Zhang

k.zhang-1@tudelft.nl

1 Department of Biomechanical Engineering, Faculty of Mechanical, Maritime and Materials Engineering, Delft University of Technology, Delft, The Netherlands

2 Department of Precision and Microsystems Engineering, Faculty of Mechanical, Maritime and Materials Engineering, Delft University of Technology, Delft, The Netherlands
Specifically, gecko adhesion relies on the fine-structure on the gecko toepads, which consists of arrays of microscale fibrils, each of which branches into nanoscale spatulas [1, 2]. This fibrillary structure makes the gecko toepads soft and deformable at various scales [3], resulting in the formation of a large contact area $[4,5]$. Additionally, as the formed contact is split up into multiple fibrils, when an individual fibril detaches, the load is redistributed over the remaining attached fibrils, inhibiting the growth of defects [6]. Importantly, when attached to a tilted or vertical substrate, the fibrils are loaded in their stiff tensile direction, preventing deformations that could lead to loss of the formed contact [3].

Inspired by the gecko toepads, over the last decades, researchers have been fabricating artificial adhesives consisting of arrays of microscale high-aspect-ratio cylindrical micropillars. Similar to the gecko, the functionality of these man-made adhesives relies on the deformability of the micropillars, which facilitates large contact formation, and the splitting-up of the formed contact, which inhibits the propagation of defects [6]. Detachment of cylindrical micropillars in the normal (pull-off) direction is caused by 
peak stresses developing at the edge of the contact when loads are applied, followed by the initiation of a crack that propagates from the edge towards the center of the contact [7]. The pull-off strength of individual micropillars can be increased by topping them with a thin terminal disc, resulting in so-called mushroom-shaped micropillars [8]. Pulling off mushroom-shaped pillars occurs via a thin-film peeling mechanism of the disc, directed from the center to the edge of the contact, which leads to higher pull-off forces than adhesives with straight micropillars [9].

Under shear loading, due to their high aspect ratio, micropillars tend to buckle, which drastically reduces contact area, leading to loss of grip [10,11]. Moreover, buckling causes pillars to attach to one another, which limits the reusability of the adhesive $[10,12]$. The presence of thin terminal disk, as in mushroom-shaped micropillars, cannot prevent this buckling and subsequent loss of contact [13]. Higher friction forces have been reported for adhesives topped with a thin terminal film, thanks to the high peeling strength of the terminal layer, which results in a crack trapping mechanism $[14,15]$, and adhesion hysteresis of the terminal layer [16]. Additionally, it has been hypothesized that internal sliding of micropillars underneath the terminal layer contributes to friction [16]. Tian et al. found that embedding microparticles in micropillars led to an increase in the stiffness of the micropillars, and consequently higher friction when particles were distributed homogeneously [17]. Another approach to increase friction in micropillar adhesives was reported by Bae et al. who fabricated composite mushroom-shaped micropillars with a stiff core and a soft shell [10]. The soft shell and a terminal disc allowed for firm grip, while the stiff core prevented irreversible buckling of the micropillars [10]. Durability testing of these adhesives showed that the friction performance of the reinforced adhesives over 100 of friction cycles did not decline, opposite to micropillars without reinforcement, the friction of which reduced significantly after about 15 cycles of testing [10]. Similarly, Xue et al. fabricated composite soft micropillars reinforced with bundles of stiff nanopillars and found that these reinforcements had a positive effect on the generated (dynamic) friction [18]. Minsky and Turner [19] fabricated and experimentally tested single pillars with a hard core and soft shell and reported that these composite pillars generated nine times higher adhesion and seven times higher friction than homogeneous pillars. A different reinforcement method has been proposed by Fischer et al. [20] and Gorumlu and Aksak [21], who fabricated micropillars with a stiff stem and a soft tip and showed that the adhesion of these composites deteriorated less with an increasing substrate roughness as compared to homogeneous micropillars of the same dimensions.

The studies described above on pull-off and friction forces of bio-inspired micropillar adhesives has all been done on hard substrates. Limited research has been conducted on investigating how to achieve high friction on deformable substrates by micropillar adhesives. Friction depends on the stiffness of the substrate, with a lower Young's modulus leading to better contact formation [22]. On the other hand, mechanical interlocking of patterned adhesives with substrate asperities, a mechanism that increases with increasing substrate stiffness, has been shown to be an important contributor to friction [23]. The above studies on hard substrates all indicate that the generation of firm, repetitive grip requires anisotropic mechanical properties of adhesives, that is, high deformability in the normal direction to maximize contact formation, and high stiffness in the loading (either shear or pull-off direction) to preserve the formed contact when loads are applied. In this paper, we aimed to investigate whether such stiffness anisotropy is also beneficial for generating high friction on deformable substrates.

We explored an approach of generating adhesives with high deformability in the normal direction and high stiffness in the shearing direction by combining two strategies: reinforcement of soft individual micropillars with a hard core and incorporation of bridges between neighboring micropillars. We measured pull-off and friction stresses (i.e., forces divided by the effective contact area) of the reinforced adhesives on rigid glass substrates and elastomeric poly-dimethoxysiloxane (PDMS) substrates, and compared these with the corresponding stresses generated by reference micropillar arrays without a soft shell and without interpillar bridges.

\section{Experimental}

\subsection{Fabrication methods}

\subsubsection{Adhesives}

Adhesives with bridged micropillars were fabricated in a three-step process: fabrication of a soft mold, fabrication of micropillar arrays by soft molding, and bridging of the micropillars via a spin coating process.

First, a soft mold was fabricated by replicating a patterned silicon wafer via a double-molding process. Thereto, a silanized silicon wafer with $1.0 \times 1.0 \mathrm{~cm}^{2}$ orthogonal patterns, consisting of circular pits with a diameter of $40 \mu \mathrm{m}$, a depth of $55 \mu \mathrm{m}$, and a spacing of $10 \mu \mathrm{m}$, was casted with a degassed mixture of polydimethylsiloxane base and curing agent (10:1 ratio; PDMS, Sylgard 184, Dow Corning) and cured at $70{ }^{\circ} \mathrm{C}$ for $24 \mathrm{~h}$. The as-formed PDMS micropillar array was then treated with oxygen plasma for 3 min and silanized with trichloro $(1 \mathrm{H}, 1 \mathrm{H}, 2 \mathrm{H}, 2 \mathrm{H}$-perfluorooctyl)silane (Sigma-Aldrich) in an evacuated desiccator for $2 \mathrm{~h}$. Subsequently, a degassed mixture of PDMS base and curing agent 
(10:1 ratio; Young's modulus: $580 \mathrm{kPa}$ [24]) was poured onto the modified PDMS micropillar array and cured at $70{ }^{\circ} \mathrm{C}$ for $24 \mathrm{~h}$ to obtain the wafer replica that acted as a soft mold.

In the second step, the soft mold was used to fabricate adhesives with micropillars. To facilitate demolding, the obtained soft mold was silanized with trichloro $(1 \mathrm{H}, 1 \mathrm{H}, 2 \mathrm{H}, 2 \mathrm{H}$-perfluorooctyl)silane (SigmaAldrich) in an evacuated desiccator for $2 \mathrm{~h}$. Afterwards, a custom-made aluminum frame with a square hole of $7.5 \times 7.5 \mathrm{~mm}^{2}$ and a depth of $2 \mathrm{~mm}$ was placed on the soft mold. PDMS mixtures with a 5:1 ratio of base and curing agent (Young's modulus: $1000 \mathrm{kPa}$ [24]) were degassed and cured in this frame, resulting in adhesives with micropillars with a backing layer thickness of $2 \mathrm{~mm}$, corresponding to the depth of the frame. The curing temperature and time were $70{ }^{\circ} \mathrm{C}$ and $24 \mathrm{~h}$, respectively.

In the third step, an elastomeric coating and bridges were introduced to the micropillar arrays via a spin coating procedure (Fig. 1). Specifically, PDMS with a 20:1 ratio of base and curing agent (Young's modulus: $280 \mathrm{kPa}$ [24]) was first dissolved in $45^{\circ} \mathrm{C}$ tert-butyl alcohol (TBA, Sigma-Aldrich) to control the concentration of PDMS in the solution. TBA was used as a solvent here, because it provides not only a good solubility for uncured PDMS, but also negligible swelling for cross-linked PDMS [25], preventing unwanted expansion or deformation of the micropillars. We used $5 \mathrm{wt} \%$ and $10 \mathrm{wt} \%$ of uncured PDMS in TBA as polymer solution to

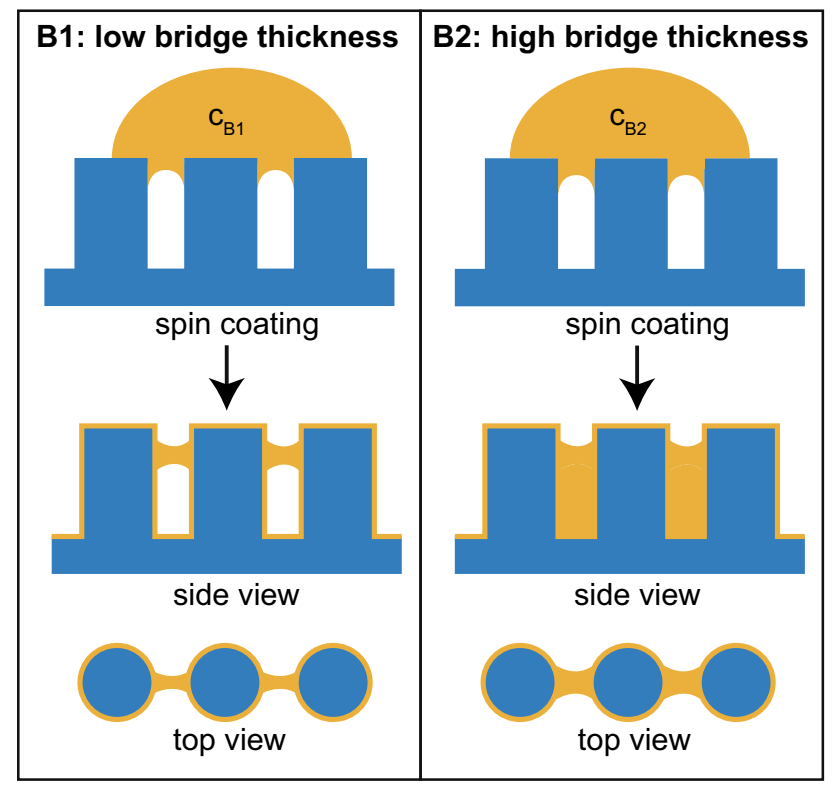

Fig. 1 Schematic of bridge fabrication. Bridges and coatings of different thickness were obtained by using spin coating precursors (yellow in the figure) with different concentration $\left(\mathrm{c}_{\mathrm{B} 1}\right.$ and $\left.\mathrm{c}_{\mathrm{B} 2}\right)$. Capillary action determines the shape and thickness of the bridges and thickness of the coating, and curing fixates the shapes of the bridges spin-coat micropillar arrays. A drop of the according polymer solution $(\sim 80 \mu \mathrm{L})$ was spin coated on the micropillars at $2000 \mathrm{rpm}$ for $3 \mathrm{~min}$, resulting in coating of the micropillars and formation of microdroplets in between neighboring micropillars. Subsequently heating of spin-coated micropillar arrays in the oven at $70{ }^{\circ} \mathrm{C}$ for $24 \mathrm{~h}$ rendered elastomeric bridges between neighboring micropillars. A polymer solution of $5 \mathrm{wt} \%$ PDMS in TBA rendered bridges with small thickness (referred henceforth to as B1), and spin-coating with $10 \mathrm{wt} \%$ PDMS in TBA resulted in bridges with large thickness (referred henceforth to as B2).

Adhesives of non-bridged micropillars, referred henceforth to as B0, were fabricated using the same method, but without the third step of spin coating. Unpatterned adhesives (UNP) were fabricated by placing the aluminum frame on a silanized glass slide, filling the frame with PDMS, and subsequent curing.

The fabricated adhesives were inspected with scanning electron microscopy (SEM, JEOL 6010) and optical microscopy (Nikon E400POL). The pillar heights and diameters before and after spin coating were determined from SEM images, using ImageJ software. Twelve micropillars were measured for each sample. The number of bridges that single micropillars were connected to was counted in a $15 \times 15$ pillar area. Three specimens, namely 675 pillars in total, were investigated for both B1 and B2.

\subsubsection{Substrates}

Adhesion and friction were measured on two types of spherical probes: from glass and from PDMS. The glass probe was a UV Fused Silica PlanoConvex Lense with a curvature radius of $46.0 \mathrm{~mm}$ and a center thickness of $3.8 \mathrm{~mm}$ (Thorlabs Inc., Newton, NJ, USA, LA4380) and was used to replicate the PDMS probe via a double-molding process. In short, the glass probe was placed in a plastic Petri dish and casted with uncured and degassed PDMS (10:1 base and curing agent mixing ratio). After curing, the formed PDMS mold was silanized and filled with uncured PDMS (10:1 base and curing agent mixing ratio), followed by degassing and curing to obtain a spherical PDMS probe.

\subsection{Friction and adhesion measurements}

\subsubsection{Measurement setup}

Friction and adhesion measurements were performed by placing the adhesive sample on a force platform, with its surface facing upwards. The spherical probe of glass or PDMS was brought from above in contact with the adhesive using a 2D translation stage PT1/M-Z8 (Thorlabs), controlled with KDC101 controllers (Thorlabs), via Kinesis software (Thorlabs). The force platform was an adapted version of a 
custom-made force transducer presented in earlier work [26]. The force platform was connected to its immobile environment via flat plate springs. Loading the sample normally or laterally resulted in displacements of the force platform, which were recorded with two confocal chromatic aberration sensors (CL1 MG210; Stil S.A.S.), controlled with Prima controllers (Stil) via the appurtenant CCS Manager software (Version 1.5.2.404; Stil). Observed displacements of the platform were translated to forces using the effective stiffness of the force platform. In an earlier calibration procedure, the stiffness of the force platform was estimated by separately loading it in the lateral and normal direction with $0,6,11,16,21$, and $26 \mathrm{~g}$, and measuring the displacements in both directions as a result of loading. Displacements of the platform were recorded at $1000 \mathrm{~Hz}$. Here, we adapted the setup by limiting the displacement of the force platform from three to two directions: the normal direction and the lateral direction of sliding.

\subsubsection{Adhesion and friction measurements}

When the substrate was brought in contact with the adhesive, the moment of contact formation could accurately be determined from measuring minor displacements of the force platform. When in contact, the substrate was moved downwards $3 \mu \mathrm{m}$ more, corresponding to a loading force of $60-90 \mathrm{mN}$. In adhesion measurements, this preload was maintained for $5 \mathrm{~s}$, after which the measurement started. In friction measurements, this normal load was maintained during the entire measurement. In adhesion measurements, the pull-off speed was $100 \mu \mathrm{m} / \mathrm{s}$. In friction measurements, the sliding speed was $500 \mu \mathrm{m} / \mathrm{s}$ and the travel distance was $3 \mathrm{~mm}$.

Per substrate (glass or PDMS), adhesion and friction were measured on each of the three types of fabricated adhesives, B0, B1 and B2, as well as unpatterned adhesives UNP. Two copies of each type of adhesive were used, each tested five times (resulting in a total of ten repetitions for each type of adhesive), in a randomized order. In each measurement, adhesion and friction were measured successively, where the order (adhesion-friction or friction-adhesion) was randomized. Before each measurement, the sample was cleaned with Scotch tape. The spherical probe was cleaned every 20 measurements with Scotch tape.

We also did a durability test, consisting of 99 successive friction measurements on glass. One sample of each type of adhesive was used in this so-called cycle measurements, using the same loads, sliding speed, and travel distance as in the friction measurements described above. The displacement data of every third measurement were recorded and used in further analysis.

\subsubsection{Data analysis}

Displacement data were analyzed in MATLAB R2018b. Displacement data were filtered and converted into forces using the obtained calibration data. From the obtained force-time plots, the maximum adhesive force and the static friction peak were acquired. These forces were divided by the effective area of each adhesive type $\left(56.25 \mathrm{~mm}^{2}\right.$ for UNP, and $28.27 \mathrm{~mm}^{2}$ for micropillar adhesives [i.e., B0, B1, B2]) to convert them into stresses. The resulted adhesion and friction stresses were used as measures of adhesion and friction performance, respectively.

Adhesion and friction stresses were compared between the different types of adhesives using a multiway analysis of variance (ANOVA) to test the effects of adhesive type and substrate type on the generated adhesion and friction performance. An alpha level of 0.001 was used for all statistical comparisons.

\section{Results}

\subsection{Fabrication of adhesives}

Table 1 shows the micropillar thickness and Fig. 2 shows representative SEM and optical microscopy images of the three micropillar adhesives.

Figure 3 shows that the number of bridges which single micropillars were connected to varied between zero and four. In B1, micropillars with different number of bridges were more or less equally present, whereas in B2, most micropillars had two bridges connected to them.

\subsection{Friction and adhesion measurements}

\subsubsection{Friction on glass and PDMS}

Table 2 and Fig. 4 show the friction and adhesion stresses of the four types of adhesives (UNP, B0, B1, and B2) on glass and PDMS substrates. A two-way ANOVA showed

Table 1 Mean (standard deviation in parentheses) of micropillar thickness of the three micropillar adhesives. Coating thickness on top of micropillars corresponds to the increase in height, whereas the coating thickness at the vertical sides of micropillars is half of the diameter increase

\begin{tabular}{lllll}
\hline & Height $(\mu \mathrm{m})$ & $\begin{array}{l}\text { Height } \\
\text { increase }(\mu \mathrm{m})\end{array}$ & Diameter $(\mu \mathrm{m})$ & $\begin{array}{l}\text { Diameter } \\
\text { increase } \\
(\mu \mathrm{m})\end{array}$ \\
\hline B0 & $55.9(0.4)$ & - & $41.0(0.6)$ & - \\
B1 & $58.6(0.7)$ & 2.7 & $42.8(0.7)$ & 1.8 \\
B2 & $60.3(0.9)$ & 4.4 & $42.3(0.5)$ & 1.3 \\
\hline
\end{tabular}




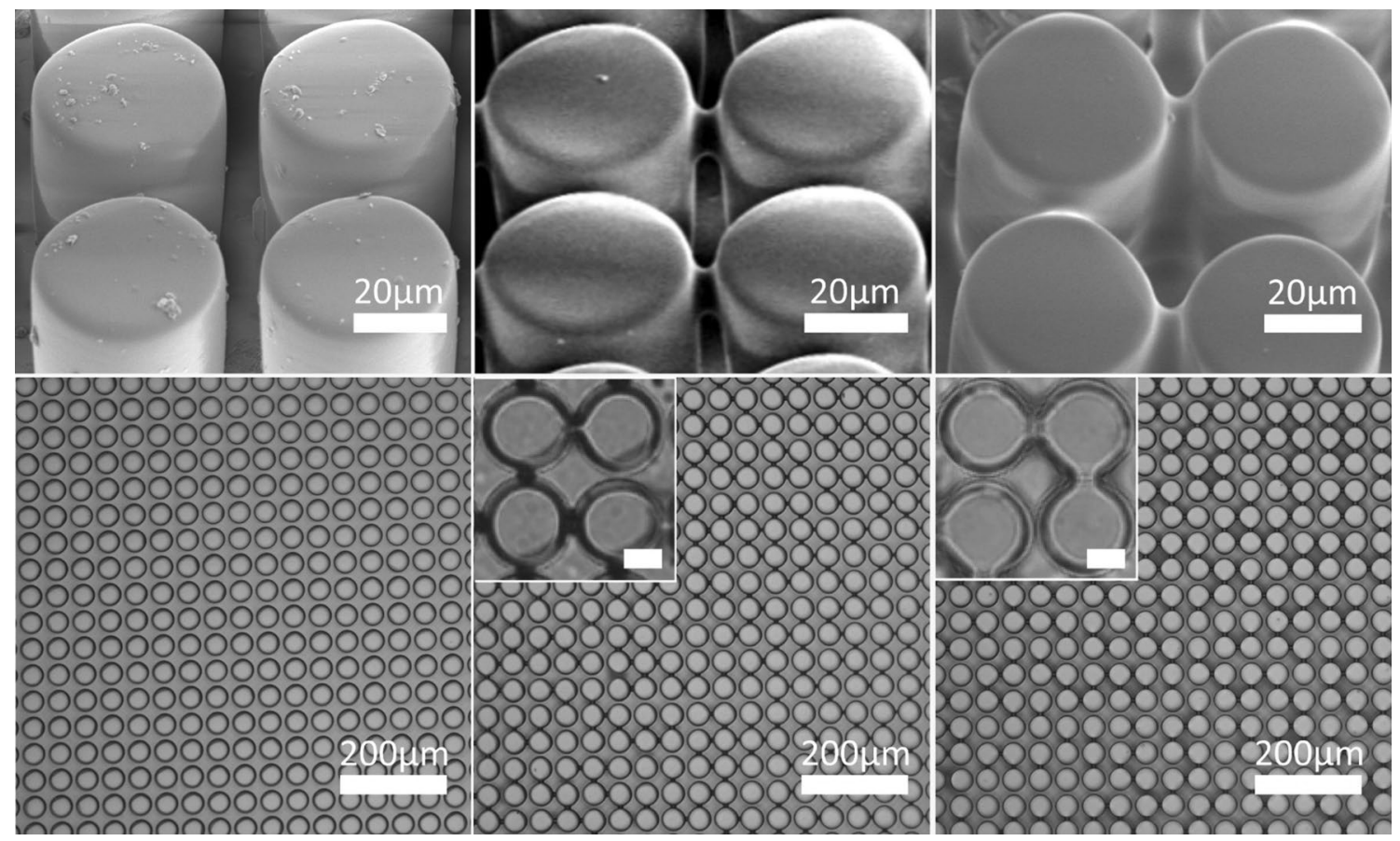

Fig. 2 SEM images (top row) and optical microscopy images (bottom row) of adhesives without bridging (B0; first column), thin bridges (B1; second column), and thick bridges (B2; third column). Scale bars of insets are $20 \mu \mathrm{m}$

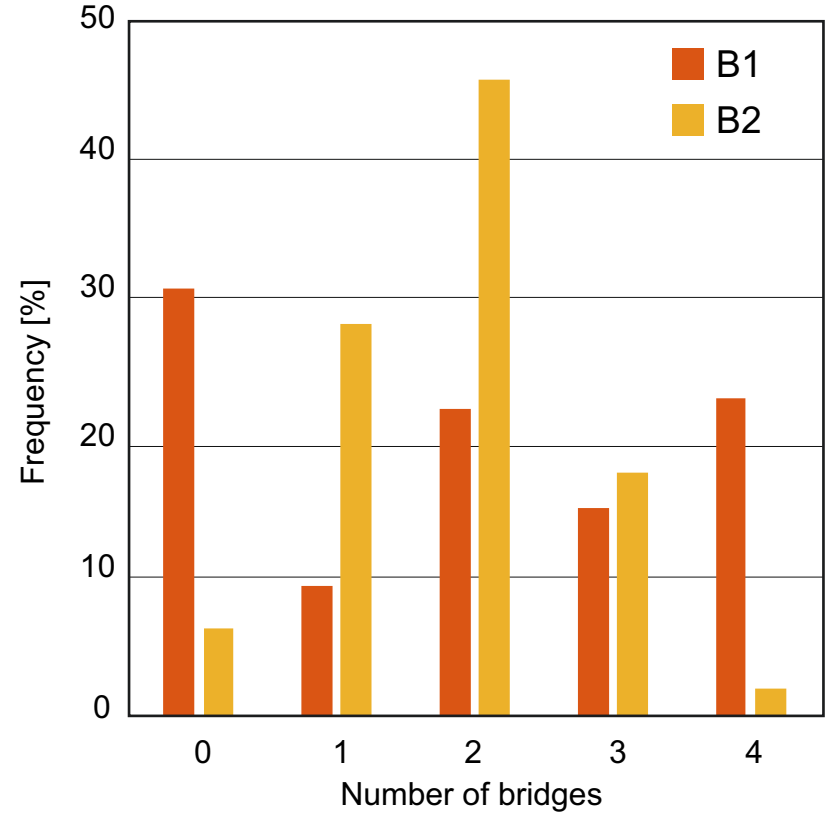

Fig. 3 Frequency of micropillars with different numbers of bridges for B1 (red) and B2 (yellow)

a significant effect of adhesive type $(F(3,156)=5.25$, $p=0.002)$ and substrate $(F(1,156)=89.0, p<0.001)$. The interaction effect between adhesive type and substrate was also significant $(F(3,156)=7.51, p<0.001)$. Post-hoc
Table 2 Mean (standard deviation in parentheses) of friction and adhesion stresses of adhesives without micropillars (UNP), and adhesives with micropillars with no bridges (B0), thin bridges (B1), or thick bridges (B2), on glass and PDMS substrates

\begin{tabular}{llllll}
\hline & \multicolumn{2}{l}{ Friction $[\mathrm{kPa}]$} & & \multicolumn{2}{l}{ Adhesion $[\mathrm{kPa}]$} \\
\cline { 2 - 3 } \cline { 6 - 6 } & Glass & PDMS & & Glass & PDMS \\
\hline UNP & $6.07(1.37)$ & $5.96(0.75)$ & & $3.03(0.31)$ & $7.03(0.86)$ \\
B0 & $3.12(0.49)$ & $5.36(0.76)$ & & $4.52(0.97)$ & $7.17(0.74)$ \\
B1 & $3.31(0.31)$ & $5.96(0.98)$ & & $3.46(0.67)$ & $5.52(0.39)$ \\
B2 & $3.52(0.32)$ & $7.06(0.75)$ & & $2.74(0.91)$ & $6.36(0.44)$ \\
\hline
\end{tabular}

analysis showed that, on glass substrates, UNP generated significantly higher friction than B0 and B1 $(p<0.001)$, whereas the difference between UNP and B2 was not statistically significant. Between the three adhesives with micropillars, no statistically significant differences were found in generated friction on glass substrates. Post-hoc analysis also showed that, on PDMS, B2 outperformed B0 and UNP $(p<0.001)$. The difference between the two adhesives with bridged micropillars, B1 and B2, was not statistically significant. Adhesives with micropillars (B0, B1, and B2) generated statistically significantly higher friction forces on PDMS than on glass $(p<0.001)$. 


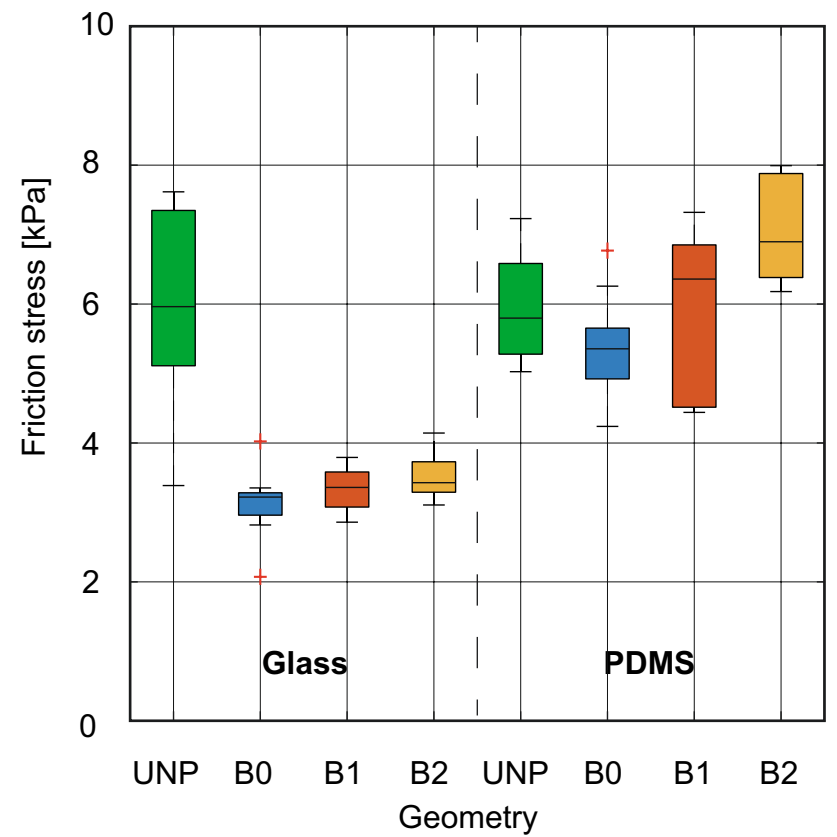

Fig. 4 Friction stress of unpatterned reference samples UNP, and the three micropillar adhesives B0, B1, and B2, on glass and PDMS substrates

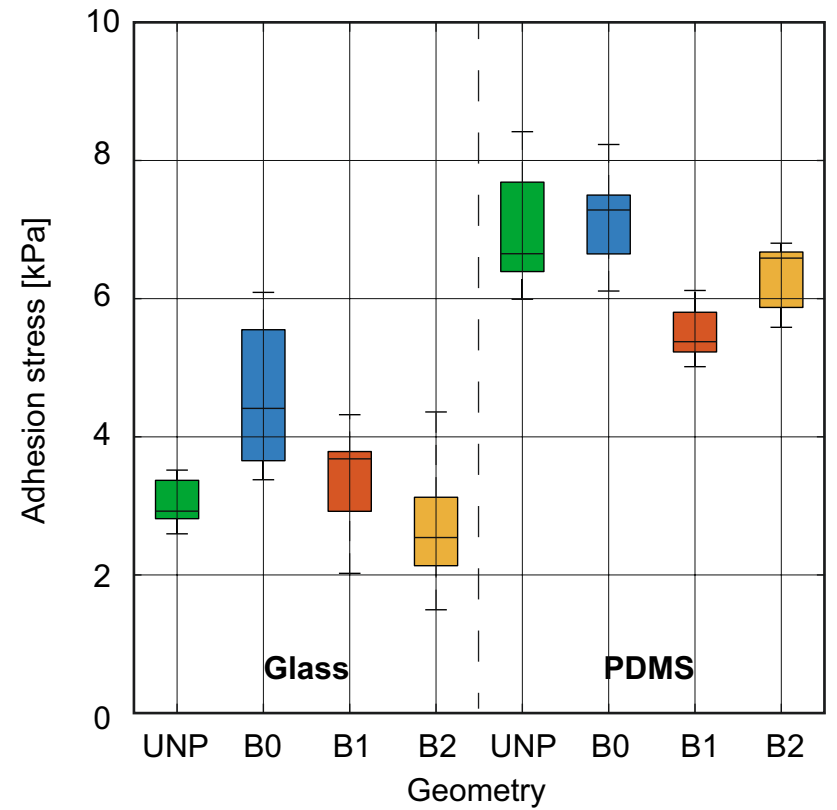

Fig. 5 Adhesion force of unpatterned reference samples UNP, and the three micropillar adhesives B0, B1, and B2, on glass and PDMS substrates

\subsubsection{Adhesion on glass and PDMS}

Figure 5 shows the adhesion stress of the four types of adhesives (UNP, B1, B2, and B3) on glass and PDMS substrates. A two-way ANOVA showed a significant effect of adhesive type $(F(3,159)=20.5, p<0.001)$ and substrate $(F(1,159)=284, p<0.001)$. An interaction effect between adhesive geometry and substrate was not observed $(F(3,159)=2.65, p=0.051)$. Post-hoc analysis showed that, on glass substrates, B0 generated statistically significantly higher adhesive stresses than $\mathrm{B} 1, \mathrm{~B} 2$, and UNP $(p<0.001)$. On PDMS, adhesives with non-bridged micropillars (B0) generated significantly higher adhesion stress than both adhesives with bridged micropillars (B1 and B2) $(p<0.001)$. All adhesive types generated significantly higher adhesive stresses on PDMS than on glass $(p<0.001)$.

\subsection{Durability of friction}

Figure 6 shows the generated peak friction forces of adhesives subjected to 99 subsequent friction force measurements on glass. The decrease of friction was the strongest during the first 3-10 cycles for all three adhesive types. In B2, friction properties were better preserved than in B0 (fitting slope -0.002 vs. -0.012 , respectively) and B1 (fitting slope $-0.010)$.

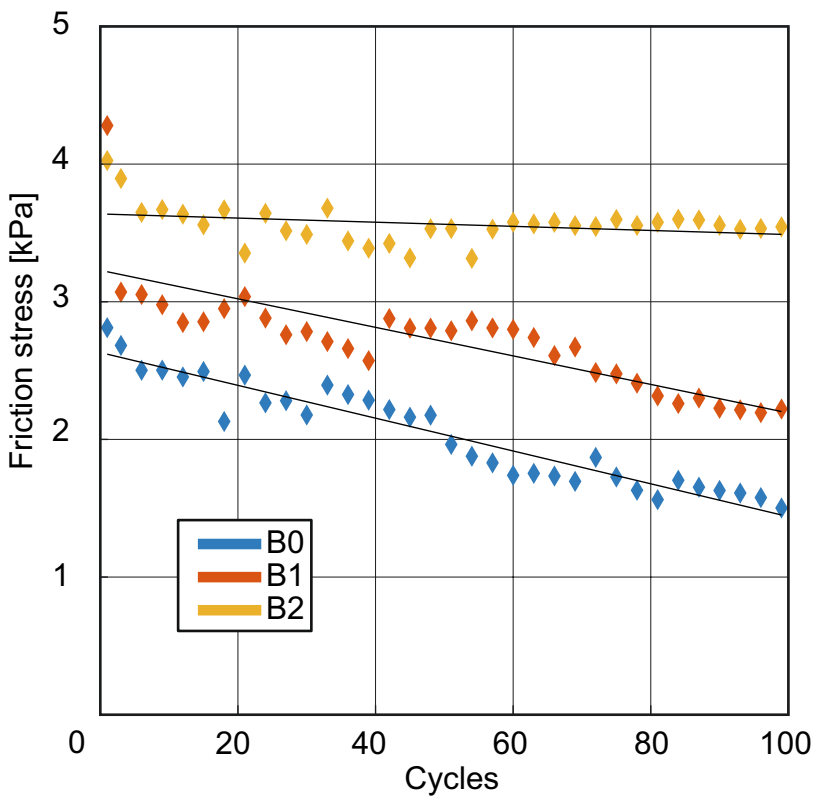

Fig. 6 Friction durability of the three micropillar adhesives (B0, B1, and B2) on a glass substrate. All three adhesive types show a steep decay in the first nine cycles 


\section{Discussion}

\subsection{Fabrication}

We showed that a relatively simple fabrication method can be used to introduce bridges in micropillar arrays, acting as lateral reinforcements. A higher concentration of PDMS in the spin-coated solution led to thicker bridges. The formation of bridges is dominated by the competition between capillary force, similar for $5 \%$ and $10 \%$ concentrations of PDMS in TBA, and structural cohesive force of the liquid bridge, increasing with increasing PDMS concentration in TBA [27]. We assume that, due to spin-coating, the polymer solution forms a film on the micropattern, and that, additionally, due to excess of polymer solution, microscale droplets are present as menisci in between neighboring micropillars. The relatively high-cohesive bridging microdroplets obtained from spin-coating with 10\% PDMS solution are larger compared to microdroplets obtained from spin-coating with 5\% DMS solution, leading to, respectively, thicker (B2) and thinner (B1) bridges after curing. Furthermore, microdroplets from a 10\% PDMS solution are presumably large enough to form two bridging microdroplets connected to a single micropillar, whereas microdroplets formed after spincoating a 5\% PDMS solution are mostly only large enough to form a single bridge in between neighboring micropillars. Consequently, the distribution of bridges (Fig. 3) is mostly clusters of two in B2 adhesives around a single micropillar, and one by one in B1 adhesives, with a random orientation. The observation that, with higher PDMS concentrations, a thicker coating is present on the top and sides of micropillars can be explained by a mechanism shown by Roy et al. who found that, when spin-coating a polymer film such as PDMS on patterned surfaces, the thickness of the polymer film increases with increasing polymer concentration [28].

\subsection{Friction measurements}

Results of friction measurements showed that, for micropillar adhesives B0, B1, and B2, significantly higher friction stresses were generated on PDMS than on glass substrates. The increased friction stresses on PDMS compared to glass substrates are partially because of substrate effects. Deformability of the PDMS substrate might introduce indentation of micropillars into the substrate, leading to interlocking [23]. Also, elastic deformations of the substrate under applied normal and lateral loads during shearing might lead to an enhanced adhesive contribution to friction, a mechanism that is not present on rigid glass substrates [22].

When assessing friction on each substrate, an effect of bridging is visible on PDMS, with friction stresses generated by adhesives with thick bridges (B2) being higher

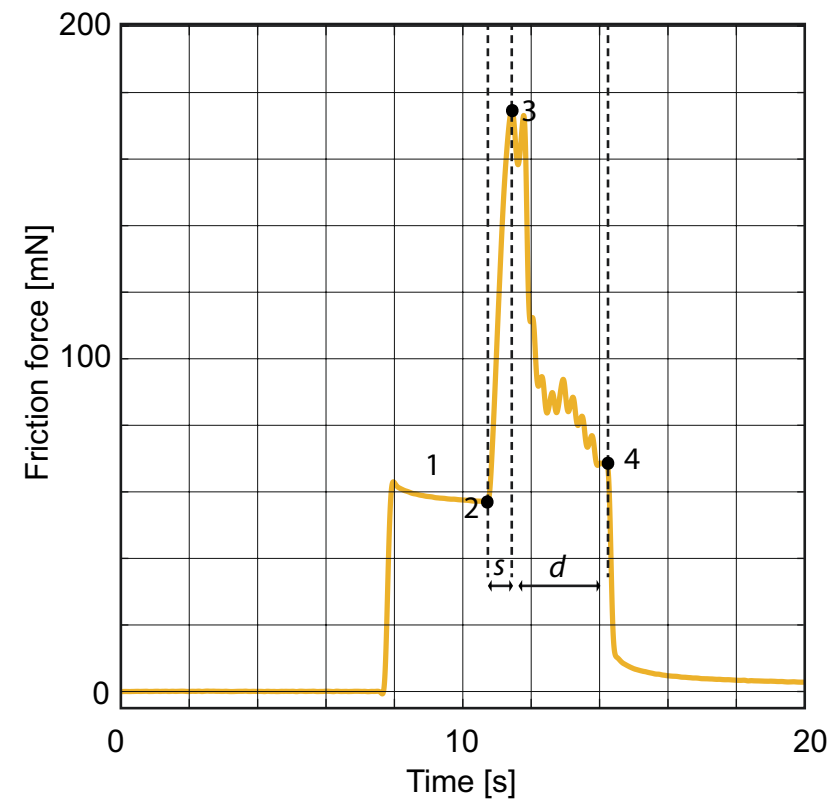

Fig. 7 Force-time plot of the tenth repetition of a micropillar adhesive with thick bridges (B2) on a PDMS substrate. At (1), the adhesive is normally loaded, resulting in a lateral load due to crosstalk of the setup. At (2), lateral loading starts. At (3), the peak static friction force has been reached, and the adhesive starts sliding. At (4), lateral loading stops, sliding ends, and friction forces drop. The timeframe between (2) and (3), annotated with $s$ in the figure, corresponds to the static friction region, and the timeframe $d$ between (3) and (4) is the dynamic friction region
Table 3 Mean (standard deviation in parentheses) of the transition time (in seconds) from static to dynamic friction for the three micropillar adhesives B0, B1, and B2, on glass and PDMS substrates

\begin{tabular}{lll}
\hline & Glass & PDMS \\
\hline B0 & $0.23(0.07)$ & $0.60(0.07)$ \\
B1 & $0.21(0.02)$ & $0.63(0.15)$ \\
B2 & $0.20(0.01)$ & $0.75(0.13)$ \\
\hline
\end{tabular}

compared to adhesives without bridges (B0). It is likely that the increased lateral stiffness of adhesives with micropillars as a result of bridging has a positive effect on interlocking on PDMS. On glass substrates, interlocking is not present, so this effect of bridging on interlocking is not observed.

Another effect of bridging is that collapsing of pillars is prevented, with preservation of contact as a result, rendering an advantage of bridged pillars (B2) over non-bridged pillars (B0) on PDMS substrates. We used force-time data of friction measurements to assess the transition from static friction, corresponding to region $s$ in the force-time plot from Fig. 7, to dynamic friction (region $d$ ). Table 3 shows the average duration of the static friction regime $s$ for $\mathrm{B} 0, \mathrm{~B} 1$, and $\mathrm{B} 2$ on glass and PDMS substrates. On glass, we found no statistically significant differences in the length of $s$ for adhesives with micropillars with or without bridging. Region $s$ was significantly longer for 
PDMS than for glass $(F(2,58)=359, p<0.001)$, illustrating that deformation of the substrate plays an important role in preservation of the contact. The length of the static friction region was not statistically significantly different between B1 and B2 on PDMS.

The effect of bridging on friction stresses being smaller on glass than on PDMS substrates might be caused by mechanical coupling between neighboring pillars on glass, which reduces contact splitting effects such as defect control and inhibition of crack growth. On deformable substrates, such as PDMS, this coupling between neighboring pillars does not necessarily lead to additional loss of contact, as the substrate might conform to the buckling pillars, resulting in preservation of contact between substrate and micropillars.

\subsection{Adhesion measurements}

The adhesive stresses we measured for micropillars without bridging on glass substrates (in the order of $4.5 \mathrm{kPa}$ ) were in same order as similar adhesives tested in literature $[14,29,30]$. In contrast to previous work which has shown hard core-soft shell structures are beneficial for adhesion [18-20, 23], our B1 and B2 adhesives exhibited similar to or lower adhesion stresses than B0, both on glass and PDMS substrates. This is not surprising, as B1 and B2 did not only had a hard core and a soft shell but also bridges. The main effect of bridging is the prevention of buckling, which hardly plays a role in adhesion experiments under the (pre)loads we applied. On glass substrates, bridging of micropillars might even be disadvantageous, as effects of contact splitting such as defect control and inhibition of crack growth are reduced by bridging. Mechanical coupling of micropillars by bridging presumably introduces a local load redistribution mechanism once one micropillar detaches, resulting in locally high peak stresses, and consequent fast defect growth. This mechanical coupling increases further with increasing bridge thickness, resulting in even lower generated adhesive stresses with adhesives consisting of micropillars with thick bridges (B2). On PDMS substrates, coupling of detachment between neighboring micropillars takes place due to deformation of the substrate under local peak stresses as described by Cheung et al. [32], even for non-bridges micropillars.

\subsection{Durability tests}

Thick bridges between micropillars led to higher durability compared to non-bridged adhesives or adhesives with thin bridges. Under shear loading of micropillar adhesives, elastic forces of bridges under compressing and stretching of bridges keep connected pillars up straight. Optical microscopic images of the samples showed that bridges were still present after the durability test and no permanent collapsed pillars were visible. When collapsed, pillars adhere to neighboring pillars, resulting in irreversible collapsing [10]. In the presence of bridges between micropillars, it is possible that bridges are elastically stretched and compressed, resulting in recovery forces on pillars as long as they are collapsed. This assumption is supported by the fact that, in the adhesion and friction measurements, where individual adhesives were measured five times, with at least $10 \mathrm{~min}$ in between measurements, no history effect was visible.

\subsection{Limitations and future work}

A limitation of our work is that we fabricated bridges with only two different shapes. It would be useful to include more concentrations of PDMS solution for explaining the effect of bridge structures on the adhesion and friction properties of micropillar adhesives. However, precisely controlling the formation of bridge structures between micropillars remains a challenge. Spin coating on a topographically patterned substrate is affected by various factors and their combinations, such as solvent properties (e.g., evaporation speed, wettability), concentration and volume of the dispensed polymer solution, and spin speed and duration. In addition, the structural characteristics of patterned micropillars, namely diameter and height of micropillars and gaps between micropillars, also influence the coating process and resultant structures. Due to this complexity, we have not been able to create samples series in which only one parameter (e.g., the bridge thickness) differs, while the rest (e.g., bridge shape) remains the same. Instead, we opted for two distinct types of bridge structures, that is, one with thinner bridges suspended between micropillars and another with thicker bridges filling the gaps. In future work, it is undoubtedly meaningful to investigate the precise formation mechanism of bridge structures, such as concentration dependence. If future work led to a thorough understanding on the relation between the settings of the spin-coating procedure and obtained bridge properties, the presented fabrication method could be a valuable addition to the currently available microfabrication toolbox [33]. Combining bridges with mushroom-shaped and spatula-shaped micropillar tips could be considered as well, for enhancing friction in particular. 


\section{Conclusion}

We fabricated composite soft micropillars with a stiff core, and introduced bridges between neighboring micropillars using spin-coating. By varying the concentration of PDMS in the spin-coated polymer solution, the geometry of the bridges, including their thickness and number density, could be varied. Friction measurements showed that bridging of micropillars has a positive effect on generated friction, presumably due to an increase in lateral stiffness and prevention of buckling, especially when attaching to deformable substrates. We also showed that in the presence of thick bridges between micropillars, the durability of friction properties of adhesives with micropillars is improved compared to adhesives with non-bridged micropillars.

Acknowledgements This research is supported by the Netherlands Organization for Scientific Research (NWO) Domain Applied and Engineering Sciences (TTW), (Open Technology Program, project 13353 "Secure and gentle grip of delicate biological tissues"). This work was also supported by a cohesion grant of the $3 \mathrm{mE}$ Faculty of Delft University of Technology between BMechE and PME departments.

Author contributions PvA and $\mathrm{KZ}$ are joint first authors, as well as corresponding authors of this manuscript. All authors contributed to the study conception and design. Fabrication and characterization were performed by KZ, and the corresponding sections in the manuscript were written by KZ. Data collection and analysis were performed by PvA, and the corresponding sections in the manuscript were written by PvA. Supervision was done by JGB and DD. All authors commented on previous versions of the manuscript. All authors read and approved the final manuscript.

Open Access This article is licensed under a Creative Commons Attribution 4.0 International License, which permits use, sharing, adaptation, distribution and reproduction in any medium or format, as long as you give appropriate credit to the original author(s) and the source, provide a link to the Creative Commons licence, and indicate if changes were made. The images or other third party material in this article are included in the article's Creative Commons licence, unless indicated otherwise in a credit line to the material. If material is not included in the article's Creative Commons licence and your intended use is not permitted by statutory regulation or exceeds the permitted use, you will need to obtain permission directly from the copyright holder. To view a copy of this licence, visit http://creativecommons.org/licenses/by/4.0/.

\section{References}

1. K. Autumn, Y.A. Liang, S.T. Hsieh, W. Zesch, W.P. Chan, T.W. Kenny, R. Fearing, R.J. Full, Nature 405, 681 (2000)

2. W. Federle, J. Exp. Biol. 209, 2611 (2006)
3. K. Autumn, J. Exp. Biol. 209, 3558 (2006)

4. K. Autumn, MRS Bull. 32, 473 (2007)

5. B.N.J. Persson, MRS Bull. 32, 486 (2007)

6. M. Kamperman, E. Kroner, A. del Campo, R.M. McMeeking, E. Arzt, Adv. Eng. Mater. 12, 335 (2010)

7. L. Afferrante, G. Carbone, Macromol. React. Eng. 7, 609 (2013)

8. S. Gorb, M. Varenberg, A. Peressadko, J. Tuma, J.R. Soc, Interface 4, 271 (2007)

9. G. Carbone, E. Pierro, S.N. Gorb, Soft Matter 7, 5545 (2011)

10. W.G. Bae, M.K. Kwak, H.E. Jeong, C. Pang, H. Jeong, K.Y. Suh, Soft Matter 9, 1422 (2013)

11. B. Murarash, M. Varenberg, Tribol. Lett. 41, 319 (2011)

12. A. Jagota, C.Y. Hui, N.J. Glassmaker, T. Tang, MRS Bull. 32, 492 (2007)

13. M. Varenberg, S. Gorb, J.R. Soc, Interface 4, 721 (2007)

14. M. Varenberg, B. Murarash, Y. Kligerman, S.N. Gorb, Appl. Phys. A Mater. Sci. Process. 103, 933 (2011)

15. N.J. Glassmaker, A. Jagota, C.-Y. Hui, W.L. Noderer, M.K. Chaudhury, Proc. Natl. Acad. Sci. 104, 10786 (2007)

16. Z. He, C.Y. Hui, B. Levrard, Y. Bai, A. Jagota, Sci. Rep. 6, 26867 (2016)

17. Y. Tian, Z. Zhao, G. Zaghi, Y. Kim, D. Zhang, R. Maboudian, A.C.S. Appl, Mater. Interfaces 7, 13232 (2015)

18. L. Xue, B. Sanz, A. Luo, K.T. Turner, X. Wang, D. Tan, R. Zhang, H. Du, M. Steinhart, C. Mijangos, M. Guttmann, M. Kappl, A. Del Campo, ACS Nano 11, 9711 (2017)

19. H.K. Minsky, K.T. Turner, A.C.S. Appl, Mater. Interfaces 9, $18322(2017)$

20. S.C.L. Fischer, E. Arzt, R. Hensel, A.C.S. Appl, Mater. Interfaces 9, 1036 (2017)

21. S. Gorumlu, B. Aksak, R. Soc, Open Sci. 4, 161105 (2017)

22. B.N.J. Persson, Tribol. Lett. 62, 34 (2016)

23. H.T. Tramsen, S.N. Gorb, H. Zhang, P. Manoonpong, Z. Dai, L. Heepe, J.R. Soc, Interface 15, 20170629 (2018)

24. J.Y. Park, S.J. Yoo, E.J. Lee, D.H. Lee, J.Y. Kim, S.H. Lee, Biochip J. 4, 230 (2010)

25. J.H. Koschwanez, R.H. Carlson, D.R. Meldrum, PLoS ONE 4, e4572 (2009)

26. P. van Assenbergh, M. Fokker, J. Langowski, J. van Esch, M. Kamperman, D. Dodou, Beilstein J. Nanotechnol. 10, 79 (2019)

27. B. Su, S. Wang, J. Ma, Y. Wu, X. Chen, Y. Song, L. Jiang, Adv. Mater. 24, 559 (2012)

28. S. Roy, K.J. Ansari, S.S.K. Jampa, P. Vutukuri, R. Mukherjee, A.C.S. Appl, Mater. Interfaces 4, 1887 (2012)

29. C. Greiner, A. Del Campo, E. Arzt, Langmuir 23, 3495 (2007)

30. D.M. Drotlef, L. Stepien, M. Kappl, W.J.P. Barnes, H.J. Butt, A. Del Campo, Adv. Funct. Mater. 23, 1137 (2013)

31. J. Jiang, Y. Wang, L. Jin, C.H. Hsu, S. Zhang, J. Mao, W. Yin, T. Li, B. Ni, Z. Su, J. Huang, C. Wesdemiotis, K. Yue, W. Zhang, S.Z.D. Cheng, A.C.S. Appl, Nano Mater. 3, 3596 (2020)

32. E. Cheung, M. Sitti, Langmuir 25, 6613 (2009)

33. P. van Assenbergh, E. Meinders, J. Geraedts, D. Dodou, Small 14, 1703401 (2018)

Publisher's Note Springer Nature remains neutral with regard to jurisdictional claims in published maps and institutional affiliations. 